\title{
An Overview of Novel and Emerging Therapies for Inflammatory Bowel Disease
}

Authors:

Disclosure:

Received:

Accepted:

Keywords:

Citation:
Sumona Bhattacharya, ${ }^{1}$ Raymond K. Cross²

1. Digestive Disease Branch, National Institute of Diabetes and Digestive and Kidney Diseases (NIDDK), National Institutes of Health (NIH), Bethesda, Maryland, USA

2. University of Maryland School of Medicine, Department of Medicine, Division of Gastroenterology and Hepatology, Baltimore, Maryland, USA

${ }^{*}$ Correspondence to rcross@som.umaryland.edu

Dr Bhattacharya has declared no conflicts of interest. Dr Cross has received income from consulting and participation in advisory boards for AbbVie, Janssen, Pfizer, Samsung Bioepis, and Takeda, outside of the submitted work.

02.07 .20

30.09 .20

Biologic therapy, Crohn's disease (CD), inflammatory bowel disease (IBD), JAK inhibition, ulcerative colitis (UC).

EMJ Gastroenterol. 2020;9[1]:91-101.

\section{Abstract}

Inflammatory bowel disease, consisting of Crohn's disease and ulcerative colitis, causes chronic gastrointestinal symptoms and can lead to morbidity and mortality if uncontrolled or untreated. However, for patients with moderate-to-severe disease, currently available therapies do not induce or maintain remission in $\mathbf{5 0} \%$ of patients. This underscores the need for additional therapies. In this review, the authors detail the novel therapies vedolizumab, tofacitinib, and ustekinumab and delve into therapies which may come onto the market within the next 10 years, including JAK-1 inhibitors (filgotinib and upadacitinib), IL-23 inhibitors (guselkumab, mirikizumab, and risankizumab), the

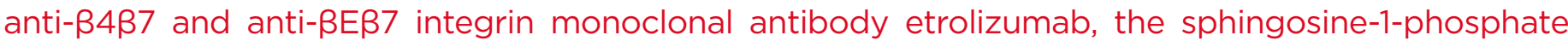
subtypes 1 and 5 modulator ozanimod, and mesenchymal stem cells. Further studies are required before these emerging therapies gain approval.

\section{INTRODUCTION}

Inflammatory bowel disease (IBD), consisting primarily of ulcerative colitis (UC) and Crohn's disease (CD), are chronic diseases affecting the gastrointestinal tract. Symptoms include diarrhoea, with and without blood, and abdominal pain. UC and CD, if left untreated or uncontrolled, cause significant morbidity and mortality at rates higher than the general population. ${ }^{1}$ Furthermore, the incidence and prevalence of IBD are increasing over time around the globe. ${ }^{2}$

Standard therapies in IBD include topical 5-aminosalicylate products such as mesalamine and sulfasalazine, thiopurines such as azathioprine and 6-mercaptopurine, and topical and systemic corticosteroids. Unfortunately, topical 5-aminosalicylate products are not recommended in moderate-to-severe UC because of a lack of efficacy in this cohort. ${ }^{3}$ Thiopurines are only effective in about onequarter of patients, ${ }^{4}$ and corticosteroids are not recommended for maintenance of remission as a result of dose-dependent short and long-term adverse effects, such as increased risk of serious infection, weight gain, elevated blood sugar levels, bone loss, and cataracts. ${ }^{5,6}$ 
Novel medications for IBD include biologics such as the anti-TNF agents infliximab and

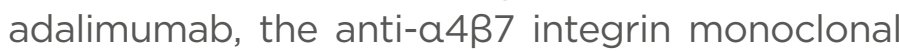
antibody vedolizumab, the anti-IL-12/IL-23 monoclonal antibody ustekinumab, and small molecules inhibiting JAK such as tofacitinib. However, in patients with moderate-to-severe $U C$ and $C D$, these medications have an average induction of remission and maintenance of remission rates $<50 \%$, ${ }^{7-13}$ underscoring the need for additional therapies for nonresponders and those who lose response to treatment. These remission rates are even lower in patients with prior exposure to biologic therapy. In this paper, the authors discuss select new IBD therapies and emerging therapies that will likely come onto the market in the next 5-10 years (Table 1). ${ }^{8-26}$

\section{NOVEL THERAPIES}

\section{Vedolizumab}

Vedolizumab is an anti-a4ß7 integrin monoclonal antibody that modulates gastrointestinal tract inflammation by inhibiting adhesion of peripheral blood lymphocytes to MAdCAM-1. ${ }^{27}$ Because of this molecule's specificity to the gastrointestinal tract, the risk of progressive multifocal leukoencephalopathy (PML) is markedly decreased compared to that of natalizumab, an anti-a-4-integrin monoclonal antibody that causes systemic immunosuppression. ${ }^{27}$

In the Gemini 1 trial, in patients with moderateto-severe UC, patients were randomised to either vedolizumab or placebo for induction. ${ }^{8}$ In the maintenance arm, patients who responded to induction therapy were randomised to vedolizumab every 8 weeks, vedolizumab every 4 weeks, or placebo for up to 52 weeks. Higher rates of clinical response (the primary outcome) at Week 6 were seen in the vedolizumab arm than the placebo arm (47.1\% versus $25.5 \%$; $p<0.001$ ). Significantly higher rates of clinical remission were seen in the vedolizumab arms compared to placebo. Rates of serious infections were similar between the arms, including no cases of PML in either group.

The Gemini 2 and 3 trials studied vedolizumab in moderate-to-severe CD. Gemini 2 had a similar trial design as that of Gemini $1 .^{13}$ More patients on vedolizumab were in remission at Week 6 compared to placebo (14.5\% versus
6.8\%; $\mathrm{p}=0.02)$. In the maintenance arm, significantly more patients on vedolizumab were in remission at Week 52 than those on placebo. Of the most common adverse events (AE), only nasopharyngitis occurred more frequently in the vedolizumab arm. No cases of PML were reported and infections, serious infections, and serious $A E$ occurred more frequently in the vedolizumab arm.

The Gemini 3 trial focussed on patients in whom prior therapy had failed, with $76 \%$ of patients having experienced anti-TNF failure ${ }^{12}$ (whereas in Gemini 2 the number of patients who had failed these agents was limited to $50 \%$ of the total). ${ }^{13}$ A nonsignificantly increased portion of antiTNF agent nonresponders in the vedolizumab arm were in clinical remission at Week 6 versus those on placebo (15.2\% versus $12.1 \%$; $p=0.443$ ). A significantly higher number of patients were in clinical remission at Week 10 in the vedolizumab arm compared to placebo (26.6\% versus $12.1 \%$; nominal $\mathrm{p}=0.001$ ). Rates of serious infections and serious AE were similar between the vedolizumab and placebo arms. Gastrointestinal infections occurred in slightly more patients in the vedolizumab arm. No cases of PML were reported.

Subcutaneous vedolizumab may be preferred by many patients given its convenience as compared to intravenous administration. In a UC trial of this formulation, patients received two doses of intravenous vedolizumab. ${ }^{28}$ Those who responded were randomised to subcutaneous vedolizumab, intravenous vedolizumab, or placebo. Significantly more patients in the subcutaneous vedolizumab arm achieved clinical remission at Week 52 than in the other two arms. Safety profiles were similar between the subcutaneous and intravenous vedolizumab arms.

Results from the study investigating subcutaneous vedolizumab in CD have only been presented in abstract form. ${ }^{29}$

\section{Tofacitinib}

Tofacitinib is a small molecule that inhibits all JAK pathways implicated in the pathogenesis of IBD, especially JAK1 and JAK3. ${ }^{30}$ Small molecules have some advantages over biologics, such as their ability to be administered orally. Furthermore, small molecules do not induce a host antibody 
response so there is no need for concomitant administration of immunomodulators (such as azathioprine, 6-mercaptopurine, or methotrexate) that carry their own toxicities and AE. Overall, these properties suggest that small molecules are easier to administer, may have a more durable response, and may have a favourable safety profile in comparison to combination of anti-TNF and immunomodulator therapy.

Table 1: Overview of ovel and emerging therapies in inflammatory bowel disease.

\begin{tabular}{|c|c|c|c|c|c|c|}
\hline Therapy & $\begin{array}{c}\text { Mechanism of } \\
\text { Action }\end{array}$ & $\begin{array}{c}\text { Route of } \\
\text { Administration }\end{array}$ & Landmark Trials & $\begin{array}{l}\text { Currently used } \\
\text { in clinical } \\
\text { practice (versus } \\
\text { investigational) }\end{array}$ & $\begin{array}{l}\text { Used in } \\
\text { Crohn's } \\
\text { disease? }\end{array}$ & $\begin{array}{l}\text { Used in } \\
\text { ulcerative } \\
\text { colitis? }\end{array}$ \\
\hline Vedolizumab & $\begin{array}{l}\text { Anti-a4ß7- } \\
\text { integrin } \\
\text { monoclonal } \\
\text { antibody }\end{array}$ & $\begin{array}{l}\text { Intravenous } \\
\text { injection, } \\
\text { subcutaneous } \\
\text { injection }\end{array}$ & $\begin{array}{l}\text { Gemini } 1^{8} \\
\text { Gemini } 2^{13} \\
\text { Gemini } 3^{12}\end{array}$ & Yes & Yes & Yes \\
\hline Tofacitinib & $\begin{array}{l}\text { JAK inhibitor } \\
\text { (preferentially } \\
\text { inhibits JAK1 and } \\
\text { JAK3) }\end{array}$ & Oral & $\begin{array}{l}\text { Octave } 1^{9} \\
\text { Octave } 2^{9} \\
\text { Octave Sustain }\end{array}$ & Yes & No & Yes \\
\hline Ustekinumab & $\begin{array}{l}\text { Anti-IL-12/-23 } \\
\text { monoclonal } \\
\text { antibody }\end{array}$ & $\begin{array}{l}\text { Intravenous } \\
\text { injection, } \\
\text { subcutaneous } \\
\text { injection }\end{array}$ & $\begin{array}{l}\text { UNITI-111 } \\
\text { UNITI-2 } \\
\text { IM-UNITI11 } \\
\text { Sands et al., }{ }^{10} 2019\end{array}$ & Yes & Yes & Yes \\
\hline Filgotinib & JAK1 inhibitor & Oral & FITZROY ${ }^{14}$ & No & Yes & No \\
\hline Upadacitinib & JAK1 inhibitor & Oral & $\begin{array}{l}\text { U-ACHIEVE }{ }^{15} \\
\text { CELEST }^{16}\end{array}$ & No & Yes & Yes \\
\hline Guselkumab & IL-23 inhibitor & $\begin{array}{l}\text { Intravenous } \\
\text { injection, } \\
\text { subcutaneous } \\
\text { injection }\end{array}$ & $\begin{array}{l}\text { None published } \\
\text { thus far }\end{array}$ & No & No & No \\
\hline Mirikizumab & IL-23 inhibitor & $\begin{array}{l}\text { Intravenous } \\
\text { injection, } \\
\text { subcutaneous } \\
\text { injection }\end{array}$ & $\begin{array}{l}\text { Sandborn et al., }{ }^{17} \\
2020\end{array}$ & No & No & Yes \\
\hline Risankizumab & IL-23 inhibitor & $\begin{array}{l}\text { Intravenous } \\
\text { injection, } \\
\text { subcutaneous } \\
\text { injection }\end{array}$ & $\begin{array}{l}\text { Feagan et al.,18 } 2017 \\
\text { Feagan et al..,19 } 2018\end{array}$ & No & Yes & No \\
\hline Etrolizumab & $\begin{array}{l}\text { Anti-a } 4 \beta 7 \text { and } \\
\text { anti-aE } \beta 7 \text { integrin } \\
\text { monoclonal } \\
\text { antibody }\end{array}$ & $\begin{array}{l}\text { Subcutaneous } \\
\text { injection }\end{array}$ & $\begin{array}{l}\text { Rutgeerts et al., }{ }^{20} \\
2013 \\
\text { EUCALYPTUS }^{21}\end{array}$ & No & No & Yes \\
\hline Ozanimod & $\begin{array}{l}\text { Sphingosine- } \\
\text { 1-phosphate } \\
\text { subtypes } 1 \text { and } 5 \\
\text { modulator } \\
\end{array}$ & Oral & TOUCHSTONE 22 & No & No & Yes \\
\hline $\begin{array}{l}\text { Mesenchymal } \\
\text { stem cells }\end{array}$ & $\begin{array}{l}\text { Differentiation } \\
\text { capacity of stem } \\
\text { cells }\end{array}$ & $\begin{array}{l}\text { Intrafistular } \\
\text { application, } \\
\text { intravenous } \\
\text { injection }\end{array}$ & $\begin{array}{l}\text { Garcia-Olmo et al., }{ }^{23} \\
2009 \\
\text { Ciccocioppo et al,, } \\
2011 \\
\text { Duijvestein et al., }{ }^{25} \\
2010 \\
\text { Forbes et al., }{ }^{26} 2014\end{array}$ & No & Yes & No \\
\hline
\end{tabular}


Results from the Octave Induction 1, Octave Induction 2, and Octave Sustain trials demonstrated the effect of tofacitinib on patients with moderate-to severe-UC.9 In the Octave Induction 1 and 2 studies, patients were randomised to receive either tofacitinib $10 \mathrm{mg}$ twice daily or placebo for 8 weeks. In the Octave Sustain trial, patients with a response to induction therapy were randomised to tofacitinib at either 5 or $10 \mathrm{mg}$ twice daily or placebo for 52 weeks. In both induction trials, tofacitinib produced a significantly higher rate of clinical remission at Week 8. Similarly, maintenance of remission at 52 weeks was significantly higher in both tofacitinib groups.

Worsening of UC, nasopharyngitis, headache, and arthralgias were the most common $\mathrm{AE}$ reported. Serious AE were not more common in the tofacitinib arms. Infection rates were higher in the tofacitinib arms in both induction and maintenance trials. Five patients who had received tofacitinib experienced cardiovascular events. One episode of gastrointestinal perforation occurred in a patient with cytomegalovirus infection on prednisone. The discovery of increased rates of pulmonary embolism and mortality in older rheumatoid arthritis patients with at least one cardiovascular risk factor receiving higher dose tofacitinib ${ }^{31}$ led the U.S. Food and Drug Administration (FDA) to issue a black box warning about tofacitinib. ${ }^{31,32}$ Tofacitinib should be reserved for patients failing biologic therapy and it is recommended that the dose is reduced to $5 \mathrm{mg}$ twice daily after successful induction with $10 \mathrm{mg}$ twice daily. Results of the effect of tofacitinib in CD have not been as promising as in UC. ${ }^{33,34}$

\section{Ustekinumab}

Ustekinumab is a monoclonal antibody against the $\mathrm{p} 40$ subunit of the $\mathrm{IL}-12 /-23$ receptors. Results from the Phase III trials (UNITI-1, UNITI-2, IM-UNITI) were published after promising results from Phase Ila and IIb trials, ${ }^{35,36}$ which consisted of an 8 week induction trial and 44 week maintenance trial in patients with moderateto-severe CD.1 The UNITI-1 trial consisted of patients who had primary nonresponse, secondary nonresponse, or AE from anti-TNF agents. The UNITI-2 trial consisted of patients who did not respond to or experience AE from immunosuppressants or glucocorticoids. In both the UNITI-1 and UNITI-2 trials, patients in either ustekinumab arm were more likely to achieve the primary endpoint (clinical remission at Week 6) compared to placebo. Similarly, in the IM-UNITI trial, more patients on ustekinumab were in clinical remission at Week 44, compared to patients on placebo. Rates of $A E$, including serious $A E$, were similar across arms in the induction and maintenance trials. Long-term extension trial results have been similarly promising. ${ }^{37}$

In an 8 week induction trial studying ustekinumab in UC, patients were assigned either fixed or weight-based doses of ustekinumab or placebo. ${ }^{10}$ Patients that responded to ustekinumab were then randomised to one of two frequencies of ustekinumab or placebo in the 44-week maintenance trial. Patients in both ustekinumab arms of the induction trial were more likely to achieve clinical remission than those given placebo ( $p<0.001$ for both comparisons). Patients in both ustekinumab arms of the maintenance trial were significantly more likely to achieve clinical remission than those in the placebo arm. In the induction trial, $A E$ were similar across all three arms, with serious AE highest in the placebo arm. In the maintenance trial, higher rates of $A E$, including serious $A E$, were reported in the placebo arm.

\section{EMERGING THERAPIES}

\section{JAK1 Inhibitors}

\section{Filgotinib}

Whereas tofacitinib inhibits all JAK with a preference for JAK1 and JAK3,30 filgotinib selectively inhibits JAK1 only. ${ }^{38}$ In the Phase II FITZROY study, the safety and efficacy of filgotinib was tested in patients with moderateto-severe $C D .{ }^{14}$ In the first 10 weeks, patients were randomised to either filgotinib or placebo. In the following 10 weeks, patients were stratified based on prior clinical response, prior anti-TNF agent exposure, and baseline corticosteroid use, among other considerations, to one of two doses of filgotinib or placebo. More patients in the filgotinib group achieved clinical remission than those in the placebo group in the induction phase of the trial (47\% versus 23\%; $p=0.0077$ ). $A E$ were similar between treatment and placebo arms, but serious $A E$ occurred at higher rates 
in the treatment arm, including four patients who developed serious infections. Additional trials studying filgotinib are underway in $C D^{39,40}$ and UC. 41,42

\section{Upadacitinib}

Upadacitinib is a JAK inhibitor that selectively binds $\mathrm{JAK}^{4}{ }^{43}$ similar to filgotinib. This small molecule has been studied in Phase II trials in both UC and $C D{ }^{15,16}$ Results from the induction trial of the Phase Ilb trial U-ACHIEVE, studied upadacitinib in moderate-to-severe UC. ${ }^{15}$ All patients in the study had inadequate response to or loss of response to corticosteroids, immunosuppressives, or biologics. The study consisted of two 8-week parts. In the first part, eligible patients were randomly assigned to receive one of four doses of oral upadacitinib or placebo. In the second part, eligible patients were randomly assigned to one of two doses of upadacitinib.

The primary endpoint of clinical remission at 8 weeks was achieved in more patients receiving upadacitinib than those receiving placebo. Of the patients receiving the $7.5 \mathrm{mg}$ dose, $8.5 \%$ achieved the primary endpoint. This increased to $14.3 \%$ in those receiving the $15 \mathrm{mg}$ dose, $13.5 \%$ receiving the $30 \mathrm{mg}$ dose, and 19.6\% receiving the $45 \mathrm{mg}$ dose, whereas $0.0 \%$ achieved clinical remission with placebo (respective $\mathrm{p}$ value comparisons with placebo: $\mathrm{p}=0.052, \mathrm{p}=0.13, \mathrm{p}=0.011$, and $\mathrm{p}=0.002$, respectively). Fewer patients who had previously failed anti-TNF agents responded to upadacitinib. More patients on upadacitinib demonstrated biologic, endoscopic, and histologic response. Rates of $A E$ were similar between patients receiving upadacitinib and placebo, including serious $A E$ and serious infections. Further studies of upadacitinib in UC are underway. ${ }^{44,45}$

Upadacitinib was studied in moderate-to-severe $C D$ in the Phase II CELEST trial. ${ }^{16}$ In the 16 -week induction trial, patients were randomised to one of five upadacitinib doses or placebo, stratified by prior anti-TNF use and endoscopic disease severity. After the induction trial, patients were rerandomised to one of two upadacitinib doses or placebo for the 36-week maintenance trial. In the induction trial, the proportion of patients achieving clinical remission, the primary endpoint, or endoscopic remission were not significantly different between groups. In the maintenance trial, numerically, more patients in the $12 \mathrm{mg}$ twice daily group were in clinical and endoscopic remission than the other groups. More AE occurred in the higher dose upadacitinib arms, but the majority were mild or moderate in severity. The most serious AE occurred in the $12 \mathrm{mg}$ twice daily arm. The most frequent $A E$ included worsening $C D$, urinary tract infection, nausea/vomiting, and headache. Further studies of upadacitinib in $C D$ are in progress. ${ }^{46-50}$

\section{IL-23 INHIBITOR}

\section{Guselkumab}

Whereas ustekinumab blocks both IL-12 and IL-23 by inhibiting their shared p40 subunit, guselkumab is a more selective antagonist, targeting the p19 subunit of IL-23.51 IL-23 activates the JAK-signal transducer and activator of transcription pathways (Figure 1).51

The Th17 pathway is subsequently activated, leading to further production of cytokines such as IL-17, IL-21, IL-22, and IL-26. Guselkumab has already demonstrated efficacy without any serious $A E$ and has been approved for moderate-to-severe plaque psoriasis. No clinical trials have been published yet on this agent; however, one published case report described a female with $C D$ who achieved deep remission with guselkumab. ${ }^{52}$ Clinical trials are underway and planned for study in UC and CD. ${ }^{53-}$ 56

\section{Mirikizumab}

Like guselkumab, mirikizumab is a monoclonal antibody that binds the p19 subunit of IL23. Mirikizumab was studied in a Phase II trial in moderate-to-severe UC.17 Patients were randomised to one of three doses of intravenous mirikizumab or placebo. Those that responded to mirikizumab at Week 12 were randomised to $200 \mathrm{mg}$ subcutaneous mirikizumab every 4 or 12 weeks through to Week 52. Those that responded to placebo at Week 12 were continued on placebo through to Week 52.

Compared to placebo, more patients in the mirikizumab arms were in clinical remission at Week 12, the primary endpoint; however, these differences were nonsignificant. Significantly more patients receiving mirikizumab had a 
clinical response by Week 12 compared to placebo. Significantly more patients in the 50 $\mathrm{mg}$ and $200 \mathrm{mg}$ arms achieved endoscopic remission by Week 12 . In the maintenance trial, by Week $52,53.7 \%$ and $39.7 \%$ of patients given mirikizumab every 4 and 12 weeks, respectively, were in clinical remission, with similar rates between biologic-naïve and biologic-exposed patients. In the induction trial, the most frequent $A E$ were nasopharyngitis, worsening of UC, and anaemia. In the maintenance trial, the most frequent $A E$ were nasopharyngitis, upper respiratory tract infection, arthralgia, and influenza. Further trials are underway studying mirikizumab in UC ${ }^{57-59}$ and CD. ${ }^{60-62}$

\section{Risankizumab}

Risankizumab also targets the p19 subunit of IL-23. A Phase II, 12-week induction study in patients with moderate-to-severe CD compared intravenous risankizumab to placebo. ${ }^{18}$ Patients were randomised to either $200 \mathrm{mg}$ or $600 \mathrm{mg}$ of intravenous risankizumab or placebo, each administered every 4 weeks. Significantly more patients in the risankizumab groups achieved clinical remission at Week 12, the primary endpoint. Higher numbers of patients in the $600 \mathrm{mg}$ compared with placebo group achieved clinical response, endoscopic remission, and deep remission. Mucosal healing, defined as absence of mucosal ulceration, was not achieved at higher rates in the risankizumab groups compared with placebo. Serious AE included worsening of $C D$ and infections ( $n=3$ [risankizumab groups]; $n=3$ [placebo group]).

Results from an open-label extension study were subsequently published. ${ }^{19}$ Of the 44 patients in the open-label extension arm, $71 \%$ were in clinical remission at Week 52 and $81 \%$ demonstrated a clinical response. Further studies on risankizumab are underway in UC $\mathrm{C}^{63,64}$ and $C D{ }^{65-67}$

\section{Anti- $\alpha 4 \beta 7$ and Anti-aeß7 Integrin Monoclonal Antibody}

\section{Etrolizumab}

Etrolizumab is a monoclonal antibody against a $4 \beta 7$ and aEB7 integrin and thereby prevents leukocyte binding with MAdCAM-1 and E-cadherin, respectively (Figure 2). ${ }^{37}$ After promising Phase I results, ${ }^{20}$ the Phase II trial (EUCALYPTUS) results were published. ${ }^{68}$



Figure 1: IL-12 and IL-23 differentiation pathways.

CD: cluster of differentiation; ILC: innate lymphoid cells; NKT: natural killer T.

From "Anti-interleukin-23 agents for the treatment of ulcerative colitis" by Jurij Hanžel \& Geert R. D'Haens, Expert Opinion on Biological Therapy, published April, 2020, reprinted by permission of the publisher (Taylor \& Francis Ltd). 




Figure 2: Etrolizumab mechanism of action.

IEL: Intraepithelial lymphocytes; MadCAM-1: mucosal addressin cell adhesion molecule 1; VCAM-1: Vascular cell adhesion protein 1.

From Sandborn et al.,37 2020. Creative Commons 4.0 International License (https://creativecommons.org/licenses/by$n c / 4.0 /$ ).

In EUCALYPTUS, patients underwent randomisation with assignment to one of two doses of subcutaneous etrolizumab or placebo.

At 10 weeks, statistically more patients in the etrolizumab arms were in clinical remission compared to the placebo arm, the primary endpoint (21\%, $10 \%$, and $0 \%$ in the $100 \mathrm{mg}$ etrolizumab, $300 \mathrm{mg}$ etrolizumab plus loading dose etrolizumab, and placebo group, respectively, [comparisons $\mathrm{p}=0.0040$ and $\mathrm{p}=0.048]$ ). No significant differences were reported for clinical remission at Week 6 , clinical response at Week 10, mucosal healing (defined as a Mayo Endoscopic Subscore $O$ or 1) at Week 10, or histopathologic disease severity score. Numerically, more AE were reported in the placebo group. One serious infection was reported in the placebo group.
Further studies are underway, including openlabel extension studies in UC (SPRUCE, ${ }^{69}$ HIBISCUS I,70 HIBISCUS ${ }^{7,},{ }^{71}$ LAUREL, ${ }^{72}$ GARDENIA, ${ }^{73}$ HICKORY, ${ }^{74}$ COTTONWOOD ${ }^{75}$ ) and CD (BERGAMOT, ${ }^{76}$ JUNIPER $\left.^{77}\right) \cdot{ }^{21}$

\section{Sphingosine-1-Phosphate Subtypes 1 and 5 Modulator}

\section{Ozanimod}

Ozanimod is an oral small molecule that modulates sphingosine-1-phosphate 1 and 5 receptor subtypes. Sphingosine-1-phosphate subtype 1 has been demonstrated to play a key role in lymphocyte trafficking; when antagonised, lymphocytes are no longer able to travel from secondary lymphoid organs into the circulation. ${ }^{78}$ The TOUCHSTONE Phase II trial studied ozanimod in moderate-to-severe UC. ${ }^{22}$ In the induction phase, patients were randomised to 
one of two doses of ozanimod or placebo for 8 weeks. Patients who responded in the induction trial continued, blinded, on the medication for 24 weeks. Those that did not respond during the induction trial were given the option to continue open-label treatment.

Significantly more patients in the $1 \mathrm{mg} /$ day of ozanimod arm achieved clinical remission, the primary outcome, at Week 8 compared to placebo ( $16 \%$ versus $6 \% ; \mathrm{p}=0.048$ ). Numerically, more patients in the $0.5 \mathrm{mg} /$ day of ozanimod arm achieved clinical remission at Week 8 compared to placebo ( $14 \%$ versus $6 \% ; p=0.14$ ). Significantly more patients in both ozanimod arms demonstrated mucosal healing and Mayo Endoscopic Subscore 0 or 1 at Week 8, compared to placebo. Numerically, more patients in the ozanimod arms demonstrated histologic remission at Week 8 compared to placebo.

At Week 32, significantly more patients receiving $1 \mathrm{mg}$ or $0.5 \mathrm{mg} /$ day of ozanimod remained in clinical remission compared to placebo (21\%, $26 \%$, and $6 \%$, respectively; $p=0.01$ and $p=0.002$ compared to placebo). Mucosal healing and histologic remission at Week 32 were achieved in significantly more patients in both ozanimod arms compared to placebo. More AE were reported in the placebo arm, including serious $A E$ and $A E$ leading to drug discontinuation.

Preliminary results from the Phase III trial studying ozanimod in $\mathrm{UC}^{79}$ have been reported as promising, with significantly increased numbers of patients achieving clinical remission after induction therapy at Week 10 and maintaining remission up to Week 52;80 published results are pending. Further studies are underway with ozanimod in UC ${ }^{81-84}$ and $C D .85,86$

\section{Mesenchymal Stem Cells}

Mesenchymal stem cells (MSC) are adult stem cells which lack the immunogenicity required for preconditioning regimens. Trials have been performed using intrafistular autologous adipose-derived stem cells (ASC), ${ }^{23}$ intrafistular bone marrow-derived mesenchymal stem cells (bmMSC), ${ }^{24}$ and intravenous bmMSC in both fistulising ${ }^{25}$ and luminal $C D^{26}$ with mixed results.
Numerous future studies are underway to evaluate the role of MSC in both CD and UC..$^{87-90}$

\section{CONCLUSION}

Here, the authors have given an overview of novel and emerging therapies for use in the management of $C D$ and UC. Given the rates of induction of remission and maintenance of remission with current therapies, future agents with new mechanisms of action are needed. It is promising that primary endpoints of clinical remission are reported in $>50 \%$ of patients with agents such as mirikizumab and guselkumab, higher rates than achieved by agents currently approved for CD and UC. One network meta-analysis analysing rheumatoid arthritis data suggests that filgotinib is more effective than tofacitinib, with upadacitinib following tofacitinib. ${ }^{91,92}$

Future studies are also required to gain additional knowledge regarding positioning of agents. Personalised medicine or matching optimal medical therapies to patients based on their individual characteristics requires further study, although some predictive characteristics are emerging..$^{93,94}$ To date, the only head-tohead trial of biologics compared vedolizumab and adalimumab (the VARSITY trial) and the authors of the study concluded that vedolizumab outperformed adalimumab. ${ }^{95}$ In addition to efficacy, the safety profiles of each medication will play a large role in the selection of agents for each patient. Targeted therapies with higher specificty are potentially safer than existing therapies; for example JAK-1 inhibitors have been compared in the rheumatology literature to tofacitinib, a nonselective JAK inhibitor. ${ }^{96}$ Furthermore, medications with gut selectivity are likely to have more acceptable long-term safety profiles. Therapies that can be topically administered, such as MSC, are likely to play a significant role in the future management of perianal fistulising disease in $C D$. Overall, these new therapies for the management of IBD are exciting and are likely to help more patients achieve induction and long-term maintenance of remission with fewer $A E$. 


\section{References}

1. Opstelten JL et al. Mortality after first hospital admission for inflammatory bowel disease: a nationwide registry linkage study. Inflamm Bowel Dis. 2019;25:1692-9.

2. Molodecky NA et al. Increasing incidence and prevalence of the inflammatory bowel diseases with time, based on systematic review. Gastroenterology. 2012;142:46-54. e42.

3. Feuerstein JD et al. AGA Clinical Practice Guidelines on the management of moderate to severe ulcerative colitis. Gastroenterology. 2020;158(5):1450-61.

4. Panaccione R et al. Combination therapy with infliximab and azathioprine is superior to monotherapy with either agent in ulcerative colitis. Gastroenterology. 2014;146(2):392-400.e3.

5. Curtis JR et al. Populationbased assessment of adverse events associated with long-term glucocorticoid use. Arthritis Rheum. 2006;55(3):420-6.

6. Lichtenstein GR et al. Serious infections and mortality in association with therapies for Crohn's disease: TREAT registry. Clin Gastroenterol Hepatol. 2006;4(5):621-30.

7. Peyrin-Biroulet L, Lémann M. Review article: remission rates achievable by current therapies for inflammatory bowel disease. Aliment Pharmacol Ther. 2011;33(8):870-9.

8. Feagan BG et al. Vedolizumab as induction and maintenance therapy for ulcerative colitis. N Engl J Med. 2013;369(8):699-710.

9. Sandborn WJ et al. Tofacitinib as induction and maintenance therapy for ulcerative colitis. N Engl J Med. 2017;376(18):1723-36.

10. Sands BE et al. Ustekinumab as induction and maintenance therapy for ulcerative colitis. N Engl J Med. 2019;381:1201-14

11. Feagan BG et al. Ustekinumab as induction and maintenance therapy for Crohn's disease. N Engl J Med. 2016;375(20):1946-60.

12. Sands BE et al. Effects of vedolizumab induction therapy for patients with Crohn's disease in whom tumor necrosis factor antagonist treatment failed. Gastroenterology. 2014;147(3):618-27.e3.

13. Sandborn WJ et al. Vedolizumab as induction and maintenance therapy for Crohn's disease. N Engl J Med. 2013;369(8):711-21.

14. Vermeire $\mathrm{S}$ et al. Clinical remission in patients with moderate-tosevere Crohn's disease treated with filgotinib (the FITZROY study): results from a Phase 2 , double-blind, randomised, placebo-controlled trial. Lancet. 2017;389(10066):266-75.
15. Sandborn WJ et al. Efficacy of upadacitinib in a randomized trial of patients with active ulcerative colitis. Gastroenterology. 2020;158(8):213949.e14.

16. Sandborn WJ et al. Efficacy and safety of upadacitinib in a randomized trial of patients with Crohn's Disease. Gastroenterology. 2020;158(8):2139-49.e14.

17. Sandborn WJ et al. Efficacy and safety of mirikizumab in a randomized Phase 2 study of patients with ulcerative colitis. Gastroenterology. 2020;158(3):53749.e10.

18. Feagan BG et al. Induction therapy with the selective interleukin-23 inhibitor risankizumab in patients with moderate-to-severe Crohn's disease: a randomised, double-blind, placebo-controlled Phase 2 study. Lancet. 2017;389(10080):1699-709.

19. Feagan BG et al. Risankizumab in patients with moderate to severe Crohn's disease: an open-label extension study. Lancet Gastroentero Hepatol. 2018;3(10):671-80.

20. Rutgeerts PJ et al. A randomised Phase I study of etrolizumab (rhuMAb $B 7)$ in moderate to severe ulcerative colitis. Gut. 2013;62:1122-30.

21. Sandborn WJ et al. Etrolizumab for the treatment of ulcerative colitis and Crohn's disease: an overview of the Phase 3 clinical program. Adv Ther. 2020;37(7):3417-31.

22. Sandborn WJ et al. Ozanimod induction and maintenance treatment for ulcerative colitis. N Engl J Med. 2016;374(18):1754-62.

23. Garcia-Olmo D et al. Expanded adipose-derived stem cells for the treatment of complex perianal fistula: a Phase II clinical trial. Dis Colon Rectum. 2009;52(1):79-86.

24. Ciccocioppo R et al. Autologous bone marrow-derived mesenchymal stromal cells in the treatment of fistulising Crohn's disease. Gut. 2011;60(6):788-98

25. Duijvestein M et al. Autologous bone marrow-derived mesenchymal stroma cell treatment for refractory luminal Crohn's disease: results of a Phase I study. Gut. 2010;59(12):1662-9.

26. Forbes GM et al. A Phase 2 study of allogeneic mesenchymal stromal cells for luminal Crohn's disease refractory to biologic therapy. Clin Gastroentero Hepatol. 2014;12(1):64-71.

27. Soler $\mathrm{D}$ et al. The binding specificity and selective antagonism of vedolizumab, an anti-alpha4beta7 integrin therapeutic antibody in development for inflammatory bowel diseases. J Pharmacol Exp Ther. 2009;330(3):864-75.

28. Sandborn WJ et al. Efficacy and safety of vedolizumab subcutaneous formulation in a randomized trial of patients with ulcerative colitis Gastroenterology. 2020;158(3):56272.e12.

29. Vermeire S et al. Efficacy and safety of vedolizumab SC in patients with moderately to severely active Crohn's disease: results of the VISIBLE 2 study. J Crohns Colitis. 2020;14(Suppl 1):SO2O-1.

30. Danese $S$ et al. JAK inhibition using tofacitinib for inflammatory bowel disease treatment: a hub for multiple inflammatory cytokines. Am J Physiol Gastrointest Liver Physiol. 2016;310(3):G155-62.

31. Sandborn WJ et al. Venous thromboembolic events in the tofacitinib ulcerative colitis clinical development programme. Aliment Pharmacol Ther. 2019;50(10):1068-76

32. U.S. Food and Drug Administration (FDA). FDA approves boxed warning about increased risk of blood clots and death with higher dose of arthritis and ulcerative colitis medicine tofacitinib (Xeljanz, Xeljanz XR). 2019. Last accessed: 26 June 2020. Available at: https://www.fda.gov/drugs/ drug-safety-and-availability/fdaapproves-boxed-warning-aboutincreased-risk-blood-clots-anddeath-higher-dose-arthritis-and.

33. Sandborn WJ et al. A Phase 2 study of tofacitinib, an oral Janus kinase inhibitor, in patients with Crohn's disease. Clin Gastroenterol Hepatol. 2014;12(9):1485-93.e2

34. Panés $\mathrm{J}$ et al. Tofacitinib for induction and maintenance therapy of Crohn's disease: results of two Phase IIb randomised placebo-controlled trials. Gut. 2017;66(6):1049-59.

35. Sandborn WJ et al. A randomized trial of Ustekinumab, a human interleukin-12/23 monoclonal antibody, in patients with moderateto-severe Crohn's disease. Gastroenterology. 2008;135(4):113041.

36. Sandborn WJ et al. Ustekinumab induction and maintenance therapy in refractory Crohn's disease. N Engl J Med. 2012;367(16):1519-28.

37. Sandborn WJ et al. Long-term efficacy and safety of ustekinumab for Crohn's disease through the second year of therapy. Aliment Pharmacol Ther. 2018;48(1):65-77.

38. Van Rompaey $L$ et al. Preclinical characterization of GLPG0634, a selective inhibitor of JAK1, for the treatment of inflammatory diseases. J Immunol. 2013;191(7):3568-77.

39. Gilead Sciences. Filgotinib in the induction and maintenance of remission in adults with moderately to severely active Crohn's disease (Diversity1). NCTO2914561. https:// clinicaltrials.gov/ct2/show/ NCTO2914561. 
40. Gilead Sciences. Filgotinib in longterm extension study of adults with Crohn's disease (DIVERSITYLTE). NCTO2914600. https://clinicaltrials. gov/ct2/show/NCTO2914600.

41. Gilead Sciences. Filgotinib in the induction and maintenance of remission in adults with moderately to severely active ulcerative colitis (SELECTION1). NCTO2914522. https://clinicaltrials.gov/ct2/show/ NCTO2914522.

42. Gilead Sciences. Filgotinib in longterm extension study of adults with ulcerative colitis (SELECTIONLTE). NCTO2914535. https://clinicaltrials. gov/ct2/show/NCT02914535.

43. Parmentier JM et al. In vitro and in vivo characterization of the JAK1 selectivity of upadacitinib (ABT-494). BMC Rheumatol. 2018;2:23

44. AbbVie. A study of the efficacy and safety of upadacitinib (ABT-494) in participants with moderately to severely active ulcerative colitis (U-Accomplish). NCTO3653026. https://clinicaltrials.gov/ct2/show/ NCT03653026.

45. AbbVie. A study to evaluate the long-term safety and efficacy of upadacitinib (ABT-494) in participants with ulcerative colitis (UC). NCT03006068. https:// clinicaltrials.gov/ct2/show/ NCT03006068.

46. AbbVie. A study of the efficacy and safety of upadacitinib (ABT-494) in participants with moderately to severely active Crohn's disease who have inadequately responded to or are intolerant to biologic therapy. NCT03345836. https://clinicaltrials. gov/ct2/show/NCT03345836.

47. AbbVie. A maintenance and longterm extension study of the efficacy and safety of upadacitinib (ABT494) in participants with Crohn's disease who completed the studies M14-431 or M14-433. NCTO3345823. https://clinicaltrials.gov/ct2/show/ NCT03345823.

48. AbbVie. A study of the efficacy and safety of upadacitnib (ABT-494) in participants with moderately to severely active Crohn's disease who have inadequately responded to or are intolerant to conventional and/ or biologic therapies. NCTO3345849. https://clinicaltrials.gov/ct2/show/ NCT03345849.

49. AbbVie. A study to evaluate the long term efficacy, safety, and tolerability of repeated administration of upadacitinib (ABT-494) in participants with Crohn's disease. NCTO2782663. https://clinicaltrials. gov/ct2/show/NCT02782663.

50. AbbVie. A study to evaluate the safety and efficacy of upadacitinib (ABT-494) for induction and maintenance therapy in participants with moderately to severely active ulcerative colitis (UC). NCTO2819635. https://clinicaltrials.gov/ct2/show/ NCTO2819635.

51. Hanžel J, D'Haens GR. Antiinterleukin-23 agents for the treatment of ulcerative colitis. Expert Opin Biol Ther. 2020;20(4):399-406.

52. Grossberg LB. A case report of successful treatment of Crohn's disease and psoriasis with guselkumab. Inflamm Bowel Dis. 2019;25(7):e84.

53. Janssen Research \& Development, LLC. A study of efficacy and safety of combination therapy with guselkumab and golimumab in participants with moderately to severely active ulcerative colitis (VEGA). NCTO3662542. https:// clinicaltrials.gov/ct2/show/ NCT03662542.

54. Janssen Research \& Development, LLC. A study of guselkumab in participants with moderately to severely active ulcerative colitis (QUASAR). NCTO4033445 https://clinicaltrials.gov/ct2/show/ NCTO4033445.

55. Janssen Research \& Development, LLC. A study of the efficacy and safety of guselkumab in participants with moderately to severely active Crohn's disease (GALAXI). NCT03466411. https://clinicaltrials. gov/ct2/show/NCT03466411.

56. Janssen Pharmaceutical K.K. A study of guselkumab in participants with moderately to severely active Crohn's disease. NCT04397263. https://clinicaltrials.gov/ct2/show/ NCTO4397263.

57. Eli Lilly and Company. A study to evaluate the long-term efficacy and safety of mirikizumab in participants with moderately to severely active ulcerative colitis (LUCENT 3). NCT03519945. https://clinicaltrials. gov/ct2/show/NCTO3519945.

58. Eli Lilly and Company. A maintenance study of mirikizumab in participants with moderately to severely active ulcerative colitis (LUCENT 2). NCT03524092. https://clinicaltrials. gov/ct2/show/NCT03524092.

59. Eli Lilly and Company. An induction study of mirikizumab in participants with moderately to severely active ulcerative colitis (LUCENT 1). NCT03518086. https://clinicaltrials. gov/ct2/show/NCT03518086.

60. Eli Lilly and Company. A study of mirikizumab (LY3074828) in participants with active Crohn's disease (SERENITY). NCTO2891226. https://clinicaltrials.gov/ct2/show/ NCTO2891226.

61. Eli Lilly and Company. A study of mirikizumab (LY3074828) in participants with Crohn's disease (VIVID-1). NCT03926130. https:// clinicaltrials.gov/ct2/show/ NCT03926130.
62. Eli Lilly and Company. A long-term extension study of mirikizumab (LY3074828) in participants with Crohn's disease (VIVID-2). NCTO4232553. https://clinicaltrials. gov/ct2/show/NCTO4232553.

63. AbbVie. A multicenter, randomized, double-blind, placebo controlled induction study to evaluate the efficacy and safety of risankizumab in subjects with moderately to severely active ulcerative colitis who have failed prior biologic therapy. NCTO3398148. https://clinicaltrials. gov/ct2/show/NCT03398148.

64. AbbVie. A study to assess the efficacy and safety of risankizumab in participants with ulcerative colitis who responded to induction treatment in M16-067 or M16-065. NCT03398135. https://clinicaltrials. gov/ct2/show/NCT03398135

65. AbbVie. A study of the efficacy and safety of risankizumab in participants with moderately to severely active Crohn's disease. NCT03105128. https://clinicaltrials.gov/ct2/show/ NCTO3105128.

66. AbbVie. A study of the efficacy and safety of risankizumab in participants with Crohn's disease. NCT03105102. https://clinicaltrials.gov/ct2/show/ NCTO3105102

67. AbbVie. A study to assess the efficacy and safety of risankizumab in participants with moderately to severely active Crohn's disease who failed prior biologic treatment. NCTO3104413. https://clinicaltrials. gov/ct2/show/NCT03104413.

68. Vermeire et al. Etrolizumab as induction therapy for ulcerative colitis: a randomised, controlled, Phase 2 trial. Lancet. 2014;384(9940):309-18

69. Genentech, Inc. Study to evaluate the long-term safety of etrolizumab in participants with moderate to severe ulcerative colitis. NCT01461317. https://clinicaltrials.gov/ct2/show/ NCT01461317.

70. Hoffmann-La Roche. A study comparing the efficacy and safety of etrolizumab with adalimumab and placebo in participants with moderate to severe ulcerative colitis (UC) in participants naive to tumor necrosis factor (TNF) inhibitors (Study \#1) (HIBISCUS I). NCTO2163659. https://clinicaltrials. gov/ct2/show/NCTO2163759.

71. Hoffmann-La Roche. A study comparing the efficacy and safety of etrolizumab with adalimumab and placebo in participants with moderate to severe ulcerative colitis (UC) in participants naive to tumor necrosis factor (TNF) inhibitors (Study \#2) (HIBISCUS II). NCTO2171429. https://clinicaltrials. gov/ct2/show/NCT02171429.

72. Hoffmann-La Roche. A study of the efficacy and safety of etrolizumab 
treatment in maintenance of disease remission in ulcerative colitis (UC) participants who are naive to tumor necrosis factor (TNF) inhibitors (LAUREL). NCTO2165215. https:// Www.clinicaltrials.gov/ct2/show/ NCTO2165215.

73. Hoffmann-La Roche. A study comparing the efficacy and safety of etrolizumab to infliximab in participants with moderate to severe ulcerative colitis who are naïve to tumor necrosis factor (TNF) inhibitors (GARDENIA). NCTO2136069. https://clinicaltrials. gov/ct2/show/NCT02136069.

74. Hoffmann-La Roche. A study of the efficacy and safety of etrolizumab in participants with ulcerative colitis who have been previously exposed to tumor necrosis factor (TNF) inhibitors (HICKORY). NCTO2100696. https://clinicaltrials.gov/ct2/show/ NCTO2100696.

75. Hoffmann-La Roche. Study for participants with ulcerative colitis previously enrolled in etrolizumab Phase II/III studies (COTTONWOOD). NCTO2118584. https://clinicaltrials. gov/ct2/show/NCT02118584.

76. Hoffmann-La Roche. A study to assess whether etrolizumab is a safe and efficacious treatment for participants with moderately to severely active Crohn's disease (BERGAMOT). NCTO2394028. https://clinicaltrials.gov/ct2/show/ NCT02394028.

77. Hoffmann-La Roche. Open-labe extension and safety study for participants with Crohn's disease previously enrolled in the etrolizumab Phase III study GA29144 (JUNIPER). NCTO2403323. https://clinicaltrials. gov/ct2/show/NCT02403323.

78. Mandala $\mathrm{S}$ et al. Alteration of Iymphocyte trafficking by sphingosine-1-phosphate receptor agonists. Science. 2002;296(5566):346-9.
79. Celgene. Safety and efficacy trial of RPC1063 for moderate to severe ulcerative colitis. NCTO2435992. https://clinicaltrials.gov/ct2/show/ NCTO2435992.

80. Bristol Myers Squibb (BMS). Bristol Myers Squibb announces positive topline results from pivotal Phase 3 true north trial evaluating zeposia (ozanimod) in patients with moderate to severe ulcerative colitis. 2020. Last accessed: 24 June 2020. Available at: https://news.bms.com/press-release/ corporatefinancial-news/bristolmyers-squibb-announces-positivetopline-results-pivota.

81. Celgene. An extension study of oral ozanimod for moderately to severely active Crohn's disease. NCT03467958. https://clinicaltrials. gov/ct2/show/NCT03467958.

82. Celgene. A placebo-controlled study of oral ozanimod as maintenance therapy for moderately to severely active Crohn's disease. NCT03464097. https://clinicaltrials. gov/ct2/show/NCT03464097.

83. Celgene. To evaluate efficacy and long-term safety of ozanimod in japanese subjects with moderately to severely active ulcerative colitis. NCT03915769. https://clinicaltrials. gov/ct2/show/NCT03915769.

84. Celgene. Open-label extension of RPC1063 as therapy for moderate to severe ulcerative colitis. NCTO2531126. https://clinicaltrials. gov/ct2/show/NCTO2531126.

85. Celgene. Induction Study \#2 of oral ozanimod as induction therapy for moderately to severely active Crohn's disease. NCT03440385. https://clinicaltrials.gov/ct2/show/ NCT03440385.

86. Celgene. Induction Study \#1 of oral ozanimod as induction therapy for moderately to severely active Crohn's disease. NCT03440372. https://clinicaltrials.gov/ct2/show/ NCT03440372.
87. The Cleveland Clinic. Mesenchymal stem cells for the treatment of pouch fistulas in Crohn's (IPAAF). NCT04073472. https://clinicaltrials. gov/ct2/show/NCT04073472.

88. Mayo Clinic. Angiographic delivery of AD-MSC for ulcerative colitis. NCTO4312113. https://clinicaltrials. gov/ct2/show/NCTO4312113.

89. University of Liege. MSC intratissular injection in Crohn disease patients (MSC). NCT03901235. https:// clinicaltrials.gov/ct2/show/ NCT03901235.

90. Mayo Clinic. Stem cell coated fistula plug in patients with Crohn's RVF. NCT03220243. https://clinicaltrials. gov/ct2/show/NCT03220243.

91. Ho Lee Y, Gyu Song G. Comparative efficacy and safety of tofacitinib. baricitinib, upadacitinib, filgotinib and peficitinib as monotherapy for active rheumatoid arthritis. J Clin Pharm Ther. 2020.45(4):674-81.

92. Biggioggero $M$ et al. Upadacitinib and filgotinib: the role of JAK1 selective inhibition in the treatment of rheumatoid arthritis. Drugs Context. 2019:8:212595.

93. Ordás I et al. Anti-TNF monoclona antibodies in inflammatory bowel disease: pharmacokinetics-based dosing paradigms. Clin Pharmacol Ther. 2012;91(4):635-46.

94. Dulai PS et al. Development and validation of a scoring system to predict outcomes of vedolizumab treatment in patients with Crohn's disease. Gastroenterology. 2018;155(3):687-95.e10.

95. Sands BE et al. Vedolizumab versus adalimumab for moderate-to-severe ulcerative colitis. N Engl J Med. 2019;381:1215-26

96. Harrington et al. JAK inhibitors in rheumatoid arthritis: an evidencebased review on the emerging clinical data. J Inflamm Res. 2020;13:519-31. 